\title{
CALET upper limits on GeV-energy gamma-ray burst emission
}

\author{
Nicholas Cannady* \\ CRESST-II/University of Maryland, Baltimore County/NASA GSFC \\ E-mail: nicholas.w.cannadyenasa.gov \\ for the CALET Collaboration ${ }^{\dagger}$
}

The CALorimetric Electron Telescope (CALET) was deployed on the International Space Station in October 2015 and has accumulated over three years of continuous data to date. The calorimeter (CAL) is sensitive to gamma rays with energies above $1 \mathrm{GeV}$, and the response has been characterized up to hundreds of $\mathrm{GeV}$. In this work we investigate counterpart GeV-energy emission to gamma-ray bursts observed by the CALET Gamma-ray Burst Monitor and the Swift and FermiGBM missions. The methodologies for the event search and the setting of upper limits in the case of non-detection are presented, along with results from the first three years of CALET operations, covering over 100 GRBs.

36th International Cosmic Ray Conference -ICRC2019-

July 24th - August 1st, 2019

Madison, WI, U.S.A.

\footnotetext{
* Speaker.

${ }^{\dagger}$ for collaboration list see PoS(ICRC2019)1177
} 


\section{Introduction}

Gamma-ray bursts (GRBs) are energetic transient events emitting over many decades of energy in the electromagnetic spectrum. GRBs are generally classified into two broad categories based on their duration ( $T_{90}$, the time over which $90 \%$ of the associated photons are detected) [1]. The classes are aptly termed short GRBs $\left(T_{90}<2 \mathrm{~s}\right.$, down to tens of ms) and long GRBs ( $T_{90}>2 \mathrm{~s}$, up to several minutes). Multi-wavelength observations showing associations with star-forming regions and optical correlations with core-collapse supernovae (e.g. [2]) suggest that long GRBs originate in the deaths of massive stars. Short GRBs are thought to be associated with compact binary mergers. This scenario is supported by the electromagnetic detection of a short GRB coincident with the gravitational wave detection of a binary neutron star merger [3].

In spite of the difference in progenitor systems, the emission at $\mathrm{GeV}$ energies is thought to arise from similar mechanisms [4]. In the case of both short and long GRBs, a relativistic jet is launched, beaming the emission along the jet axis. There exists no consensus on the physical mechanism responsible for the prompt emission. One possibility is that thermal photons produced in the fireball photosphere are reprocessed in the jet to higher energies. Other scenarios include the acceleration of electrons within the jet itself through internal shocks or magnetic reconnection events and produce synchrotron radiation. This emission could be boosted to higher energies through a synchrotron self-Compton process, whereby the photons are upscattered by the relativistic electron population. Late-time components at high energies observed after the prompt emission are thought to arise from the collision of the ejecta with the surrounding medium. An external shock is formed and the relativistic electrons lose their energy as they travel through synchrotron or bremsstrahlung emission.

Detection of GRBs at $>100 \mathrm{MeV}$ energies was achieved first by the EGRET instrument on the Compton Gamma-ray Observatory and has been dramatically improved with Fermi-LAT [5]. Observations with the LAT have revealed the presence of photons up to tens of $\mathrm{GeV}$ in some bursts. Furthermore, compared to the lower-energy emission the onset is delayed and the duration is longer. For non-detections, LAT observations have been used to infer the presence of cutoffs in the synchrotron spectrum, constraining the bulk Lorentz factor of the ejecta. The recent detection of a burst by the MAGIC telescope [6,7] at sub-TeV energies is exciting and, on publication of the MAGIC results, promises to further constrain the interaction models for high-energy GRB emission.

\section{CALET Instrumentation}

The Calorimetric Electron Telescope (CALET) [8] was launched in August 2015 and has been operational in scientific data collection on the Japanese Experiment Module Exposed Facility of the International Space Station (ISS) since October 2015. The CALET payload (shown in Figure 1) contains two main instruments: the calorimeter (CAL) and the CALET Gamma-ray Burst Monitor (CGBM; summary results at [9]). The analysis contained in this work focuses on CAL observations.

The CAL comprises three sub-detectors: the Charge Detector (CHD), the Imaging Calorimeter (IMC), and the Total Absorption Calorimeter (TASC). The CHD measures the charge of incident 


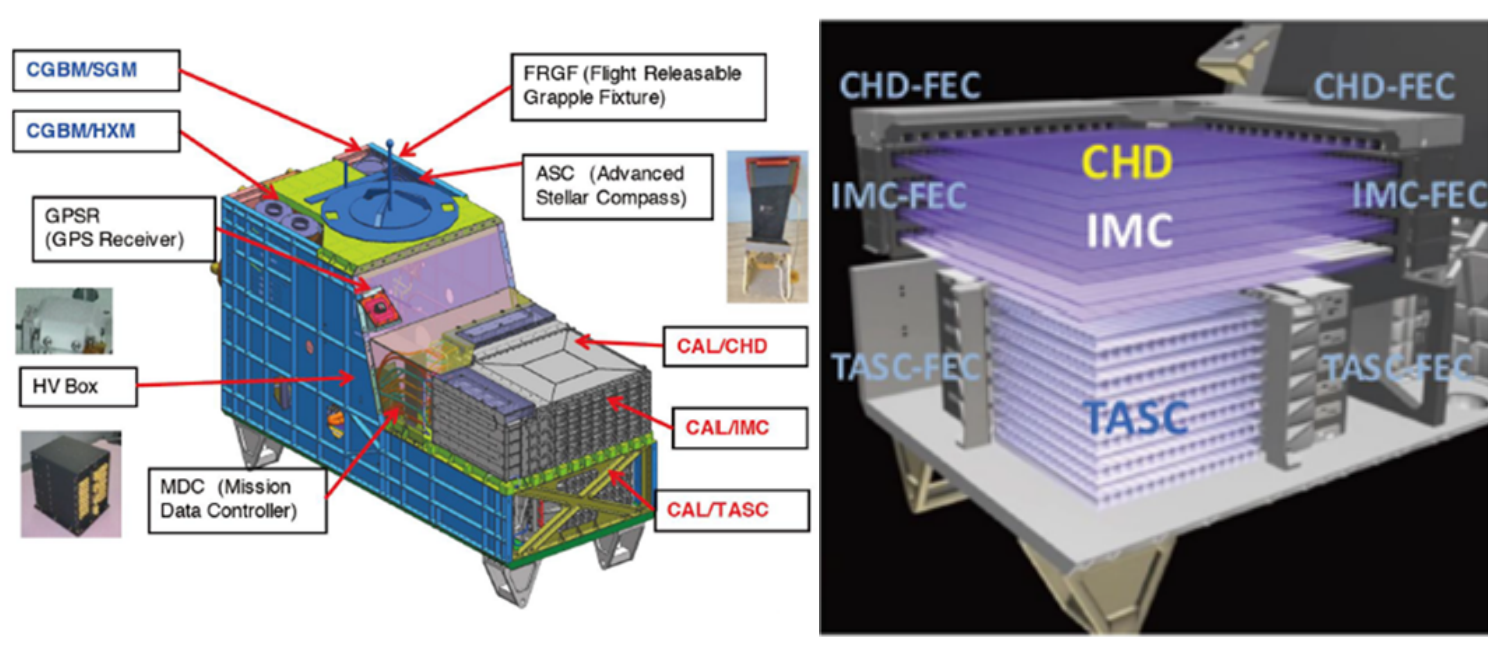

Figure 1: left: schematic of the full CALET payload. right: cutaway depiction of the CAL with the subdetectors indicated.

particles directly through ionization in a pair of crossed plastic scintillating paddles. Showers typically begin in the IMC, a sampling calorimeter (sampling fraction appr. 12\%) composed of 8 pairs of crossed plastic scintillating fiber ( $1 \mathrm{~mm} \times 1 \mathrm{~mm}$ cross section) layers, with each pair separated by tungsten sheets to stimulate shower development. The majority of the primary energy is deposited in the lead tungstate logs of the TASC, which can fully contain electromagnetic showers up to $\mathrm{TeV}$ energies. The normal incidence depth of the CAL is 30 radiation lengths $\left(X_{0} ; 3 X_{0}\right.$ in the IMC +27 $\mathrm{X}_{0}$ in the TASC). The shower image in the IMC and TASC is used to exploit the different shower topologies between hadronic and electron (or photon) primaries to achieve a proton rejection power on the order of $10^{5}[10,11]$.

Initial calibrations were performed on the ground and are regularly updated using on-orbit measurements of the instrument performance [12]. The high-energy (HE) trigger is active throughout nominal operations and has a low-energy threshold of approximately $10 \mathrm{GeV}$. A dedicated lowenergy gamma (LE- $\gamma$ ) trigger [13] is active at low magnetic latitudes (except for passage through the South Atlantic Anomaly) to reduce the low-energy threshold to approximately $1 \mathrm{GeV}$. The instrument performance for gamma-ray identification and reconstruction using the LE- $\gamma$ trigger has been optimized and characterized in detail [14]. The reconstruction of HE photon events is characterized in the same study, but optimization of the charge selection is still in progress and performance is expected to improve.

One complication arising from observing on the ISS is the obscuration of the field-of-view by external structures. A description of these complications and the background induced is described in detail in [14]. As of writing, the characterization of the moving ISS structures is improved, allowing for an increased exposure available for the analysis of photon events. The improvement is described in more detail in [15].

The sensitivity of CALET in the GeV energy range provides a potential complementary observation to that of Fermi-LAT for transient events. This sensitivity is especially valuable in the study of electromagnetic counterparts to gravitational wave events, as with the event of 2017/08/17, where GBM detection allowed a narrowing of the source region and enabled the detection of an 

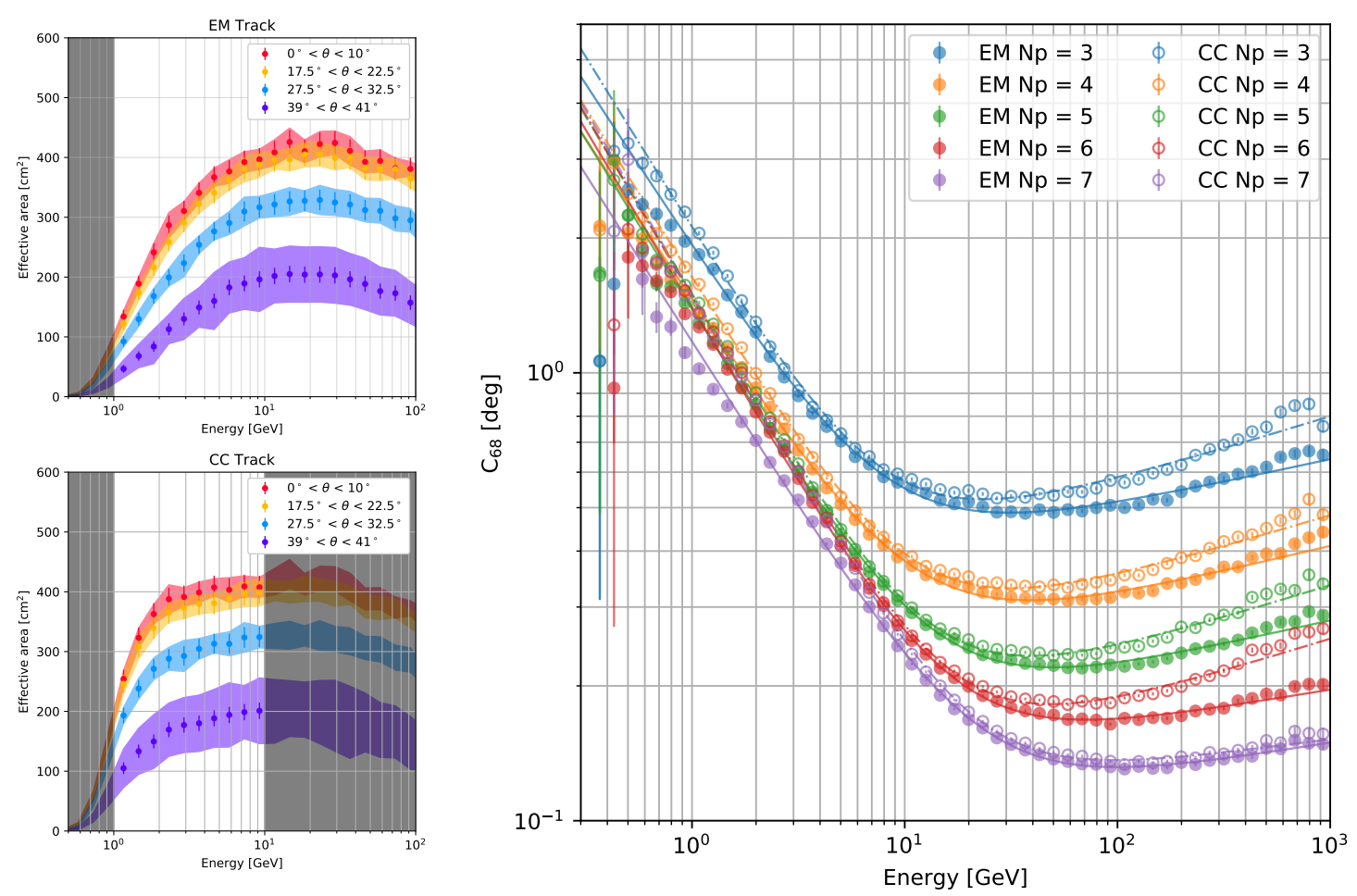

Figure 2: Effective area and angular resolution for CAL gamma-ray reconstruction [14]. CC Track and EM Track are algorithms used for the LE- $\gamma$ and HE trigger events, respectively. $\mathrm{Np}$ in the angular resolution plot corresponds to the number of layers used in the tracking, corresponding to the layer of the first pair production of the primary photon $(8-\mathrm{Np})$.

optical counterpart. The analysis presented here is similar to that performed to search for electromagnetic counterparts to gravitational waves. A full description of CALET observations up to the end of LIGO observing run $\mathrm{O} 2$ is available in $[16,17]$.

Figure 2 shows the derived effective area and angular resolution for CAL photon event reconstruction. As seen in the left panels, the CAL reaches a peak effective area of approximately $400 \mathrm{~cm}^{2}$ at a few $\mathrm{GeV}$ and maintains this level up to $50 \mathrm{GeV}$. At these energies, the charge-zero identification using the CHD fails due to the increased number of backscattered shower particles. An increase in the sensitivity at higher energies is being developed based on simulated events and flight data as previously mentioned. The angular response is parameterized in terms of the tracking algorithm (CC Track for LE- $\gamma$ trigger, EM Track for HE trigger) used and the number of layers available for the tracking. This number $\left(N_{p}\right)$ correlates with the depth of the first pair production by the primary event, with $N_{p}=3$ and $N_{p}=7$ representing pair production at the fourth and first tungsten layers, respectively. Events where $N_{p}<3$ are subject to reconstruction errors and are excluded from analysis at this stage. Events with $N_{p}=8$ are predominantly charged particles and are removed in the event selection. 


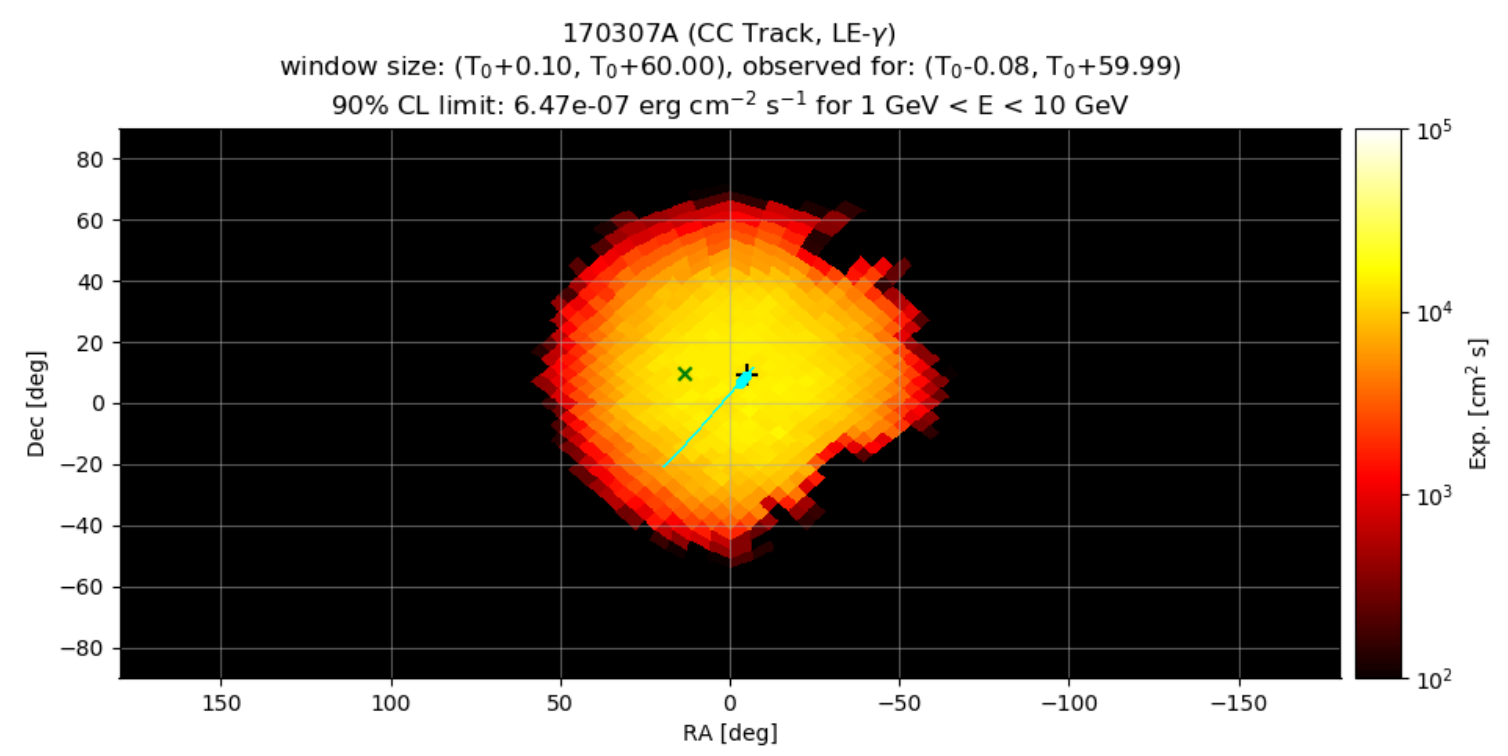

Figure 3: LE- $\gamma$ observations of GRB 170307A from the Swift catalog.

\section{Observations}

This work reflects observations covering the first three years of the CALET mission (2015/11 2018/10). We search for events in the CAL temporally and spatially consistent with GRBs reported in the archives for the Neil Gehrels Swift Observatory ${ }^{1}$ or for the Fermi Gamma-ray Burst Monitor $^{2}$. The total numbers from each catalog are 204 and 584, respectively. Of these, about 15\% were in the CAL FOV while the LE- $\gamma$ trigger was active during the prompt emission phase, but there were no CAL photon candidates within a 10 minute time window following the burst consistent with the direction reported in the table.

An example of the data products produced for the events checked is shown in Figure 3. The Swift reported position (green $\mathrm{x}$ ) for GRB 170307A was near the CAL zenith at the trigger time, shown by the black cross. The cyan line shows the path of the orbit, increasing in thickness for the time period where the LE- $\gamma$ trigger was active. The exposure is given by the pixel color on the map, and the $90 \%$ confidence level upper limit on the energy flux corresponding to the reference position is given in the bottom line of the header. For events where there are $>1 \mathrm{GeV}$ photon candidates in the time window, they are indicated on the map as well for easy vetting of spatial correlation.

\section{Sub-threshold Detection}

In addition to triggered events, the on-orbit CAL data includes counters for each trigger condition and trigger layer which are reported in the headers of each triggered event and in periodic (once per second) headers. Given the trigger rate of $10-100 \mathrm{~Hz}$ based on operational status and orbit, this provides rate data with a time resolution of $0.1-0.01$ seconds. These rate data have

\footnotetext{
${ }^{1}$ https://swift.gsfc.nasa.gov/archive/grb_table/

${ }^{2} \mathrm{https}: / /$ heasarc.gsfc.nasa.gov/W3Browse/fermi/fermigtrig.html
} 

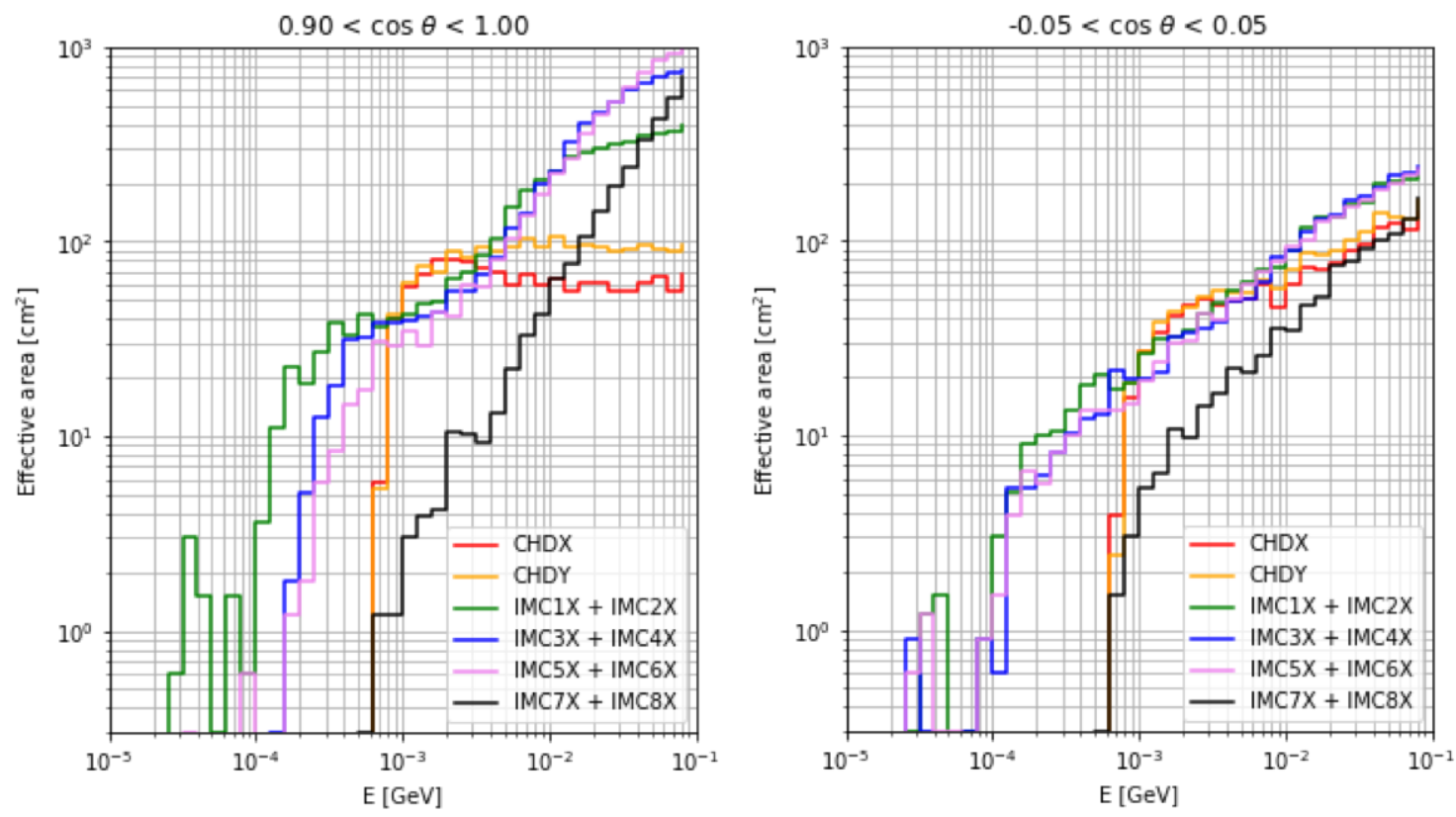

Figure 4: Preliminary effective area for the individual trigger layers in the CHD and IMC assuming the LE- $\gamma$ trigger is active.

been used to study space weather events, including relativistic electron precipitation [18] and solar energetic particles [19].

Figure 4 demonstrates the sensitivity of the CHD and IMC layers assuming the LE- $\gamma$ thresholds. Due to the periodic activation of the Single particle trigger for checking the calibration stability, these results do not have $100 \%$ duty cycle for observation. Note that these curves correspond to the summed layer signals used in the trigger logic [13]. The left and right panels show the response for photons with incident angles near zenith and near $90^{\circ}$, respectively. These are representative cases for the potential to determine spectral information through the relative strength of signals in the individual layers. The layer rates for an example event are shown in Figure 5.

\section{Summary}

We present the analysis methodology for investigation of signals from GRBs in the CALET CAL. The instrument is operating with steady data acquisition and the presence of ISS structure obstructions in the FOV have been mitigated. We followed up on triggers by the Swift and FermiGBM telescopes. At present, no significant counterpart emission has been detected at $>1 \mathrm{GeV}$, although an expansion of the analysis is in process. Upper limits are set for each event assuming a standard spectral shape and time evolution. Planned analysis will incorporate more spectral information from the individual source catalogs for comparable upper limits with the low-energy reported fluences. A search for events where spectral breaks are suggested by the lack of CAL emission is underway.

Furthermore, the potential for expanding the sensitivity of the CAL for both lower and higher energies is being explored. For low energies, the response of the trigger layers to sub-threshold 


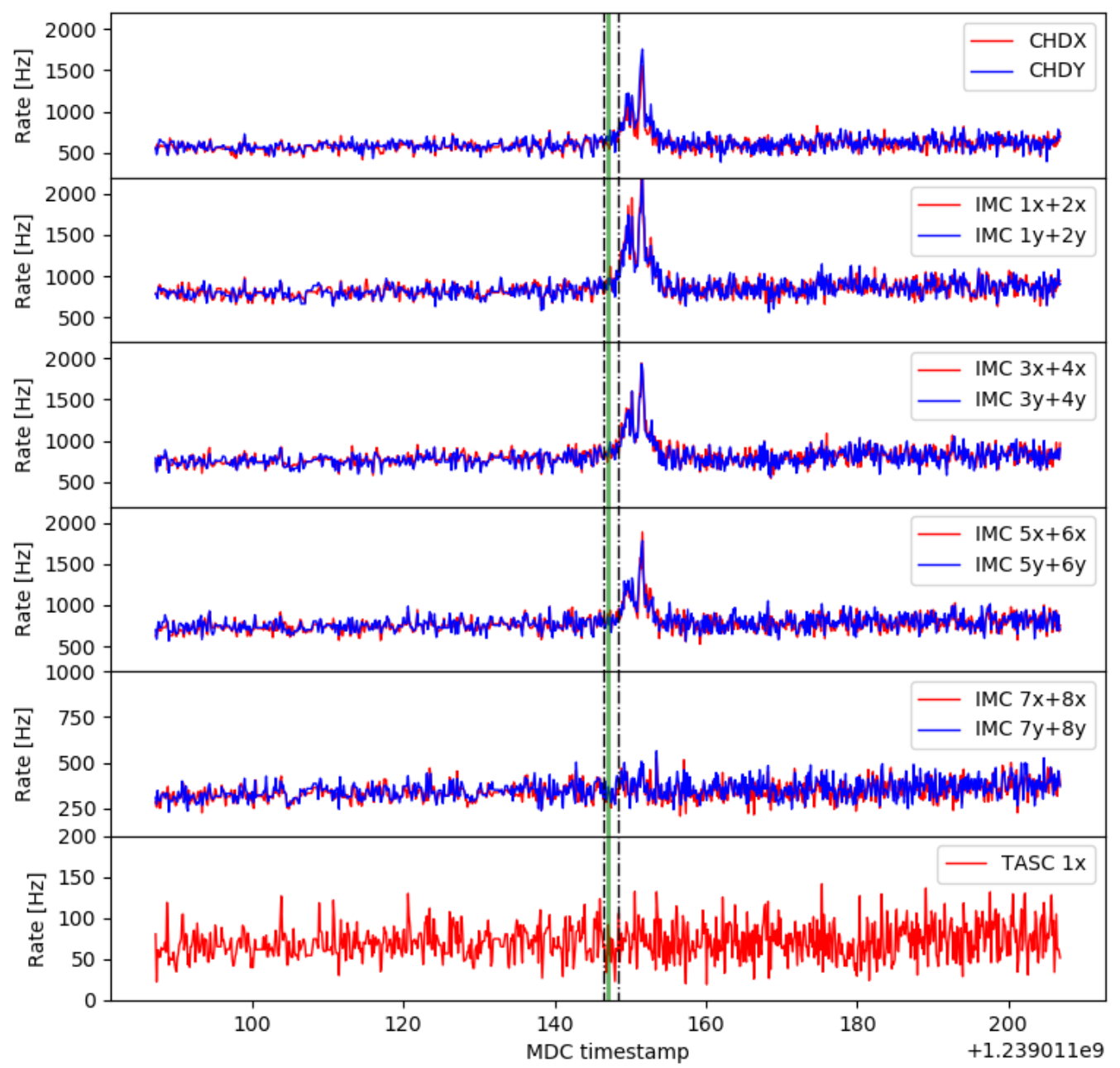

Figure 5: Individual trigger layer rates in the CAL for GRB 190411A, which was detected by CGBM. The trigger time is given by the green vertical. Dotted black vertical lines show changes in the activated triggers, although no corresponding change in thresholds occurs for this example.

photon events creates the potential for a complementary measurement with CGBM detections. Currently a polynomial characterization of the background is planned for a Bayesian blocks analysis of the rates. We are exploring the potential for estimating the distance from CALET zenith pointing using this combined information. The fine angular resolution at high energies can be exploited to use charge measurement in the upper IMC to minimize the loss due to backscattered particle contamination of the track. The use of the IMC for charge zero selection also opens the available track geometries for HE-triggered events. 


\section{References}

[1] Kouveliotou, C. et al. 1993, Identification of two classes of gamma-ray bursts, Astrophys. J. 413, L101-L104.

[2] Stanek, K. Z. et al. 2003, Spectroscopic discovery of the supernova 2003dh associated with GRB 030329, Astrophys. J. 591, L17-L20.

[3] Abbott, B. P. et al. 2018, Gravitational waves and gamma-rays from a binary neutron star merger: GW170817 and GRB 170817A, Astrophys. J. Lett. 848, L13.

[4] Nava, L. 2018, High-energy emission from gamma-ray bursts, Int. J. Mod. Phys. D 271842003.

[5] Ajello, M. et al. 2019, A decade of gamma-ray bursts observed by Fermi-LAT: The 2nd GRB catalog, arXiv:1906.11403.

[6] Mirzoyan, R. et al. 2019, GRB Coordinates Network, 23701, 1.

[7] Chand, V. et al. 2019, MAGICal GRB 190114C: cutoff in the spectrum at sub-GeV energies, arXiv: 1905.11844.

[8] Torii, S. et al. 2016, The Calorimetric Electron Telescope (CALET): a high-energy astroparticle physics observatory on the International Space Station, Proceedings of Science (ICRC 2015) 236, 581.

[9] Kawakubo, Y. et al. 2019, Gamma-ray burst observations with the CALET Gamma-ray Burst Monitor, 738 (this conference).

[10] Adriani, O. et al. 2017, Energy spectrum of cosmic-ray electron and positron from $10 \mathrm{GeV}$ to $3 \mathrm{TeV}$ observed with the Calorimetric Electron Telescope on the International Space Station, Phys. Rev. Lett. $119,181101$.

[11] Adriani, O. et al. 2018, Extended measurement of the cosmic-ray electron and positron spectrum from $11 \mathrm{GeV}$ to $4.8 \mathrm{TeV}$ with the Calorimetric Electron Telescope on the International Space Station, Phys. Rev. Lett. 120, 261102.

[12] Asaoka, Y. et al. 2017, Energy calibration of CALET onboard the International Space Station, Astropart. Phys. 91, 1-10.

[13] Asaoka, Y. et al. 2018, On-orbit operations and offline data processing of CALET onboard the ISS, Astropart. Phys. 100, 29-37.

[14] Cannady, N. et al. 2018, Characteristics and performance of the Calorimetric Electron Telescope (CALET) calorimeter for gamma-ray observations, Astrophys. J. Suppl. Ser. 238, 5.

[15] Mori, M. et al. 2019, High-energy gamma-ray observations using the Calorimetric Electron Telescope (CALET) on the ISS, 291 (this conference).

[16] Adriani, O. et al. 2016, CALET upper limits on X-ray and gamma-ray counterparts of GW151226, Astrophys. J. Lett. 829, L20.

[17] Adriani, O. et al. 2018, Search for GeV gamma-ray counterparts of gravitational wave events by CALET, Astrophys. J. 863, 160.

[18] Kataoka, R. et al. 2016, Relativistic electron precipitation at International Space Station: space weather monitoring by Calorimetric Electron Telescope, Geophys. Res. Lett. 43:9, 4119.

[19] Bruno, A. et al. 2019, Space weather observations during September 2017 with CALET on the International Space Station, 11 (this conference). 